\title{
Homoarginine, arginine, and relatives: analysis, metabolism, transport, physiology, and pathology
}

\author{
Dimitrios Tsikas $^{1} \cdot$ Guoyao $\mathrm{Wu}^{2}$
}

Received: 25 June 2015 / Accepted: 11 July 2015 / Published online: 26 July 2015

(C) Springer-Verlag Wien 2015

\begin{abstract}
The year 2008 witnessed the first report on the increase in the concentration of L-homoarginine (hArg) in the maternal plasma during human pregnancy. This observation, along with a well-known function of hArg, the methylene homologue of L-arginine (Arg), as a substrate for nitric oxide (NO) synthase, was the ignition for the start of intense research on the physiology and pathology of hArg. The circulating concentration of hArg was found to be lower in patients suffering from various diseases, and hArg emerged within only very few years as a novel cardiovascular risk factor. The compendium in hand comprises original and review articles covering several aspects of hArg, Arg and its symmetrically and asymmetrically guanidine $\left(N^{\mathrm{G}}\right)$-dimethylated derivatives SDMA and ADMA, respectively. In contrast to ADMA and SDMA, low hArg concentrations in plasma or serum and in urine are associated with high risks for morbidity and mortality, notably in the renal and cardiovascular systems. Acutely and chronically administered Arg as a nutritional supplement or in the form of dietary proteins is safe in animals and humans and leads to concomitant formation of hArg and ADMA, albeit in a different hArg/ADMA ratio. Despite the close but opposite associations of hArg and ADMA with disease in adults, children and adolescents, the underlying biochemical processes are largely unknown, presumably not restricted to NO, and warrant deeper investigation. As the common substrate for hArg and ADMA, Arg may play a
\end{abstract}

Dimitrios Tsikas

tsikas.dimitros@mh-hannover.de

1 Centre of Pharmacology and Toxicology, Hannover Medical School, Carl-Neuberg-Str. 1, 30625 Hannover, Germany

2 Department of Animal Science, Texas A\&M University, College Station, TX 77843, USA key role in the biosynthesis and homeostasis of hArg and ADMA, two putative antagonists. In animal models of stroke and obesity, hArg has beneficial effects. The potential utility of hArg as a therapeutic drug or nutritional supplement in humans and animals remains to be elaborated.

Keywords Adolescence - Adulthood AGAT ·

Biomarker $\cdot$ Childhood $\cdot$ Disease $\cdot$ Health

$\begin{array}{ll}\text { Abbreviations } \\ \text { ADMA } & \text { Asymmetric dimethylarginine } \\ \text { AGAT } & \text { Arginineglycine amidinotransferase } \\ \text { GAMT } & \text { Guanidinoacetate methyltransferase } \\ \text { GC-MS } & \text { Gas chromatography-mass spectrometry } \\ \text { GC-MS/MS } & \text { Gas chromatography-tandem mass } \\ & \text { spectrometry } \\ \text { GHD } & \text { Growth hormone deficiency } \\ \text { hArg } & \text { L-Homoarginine } \\ \text { LC-MS } & \text { Liquid chromatography-mass spectrometry } \\ \text { LC-MS/MS } & \text { Liquid chromatography-tandem mass } \\ & \text { spectrometry } \\ \text { MMA } & \text { Monomethyl arginine } \\ \text { NO } & \text { Nitric oxide } \\ \text { NOS } & \text { Nitric oxide synthase } \\ \text { OPA } & o \text {-Phthaldialdehyde } \\ \text { SDMA } & \text { Symmetric dimethylarginine }\end{array}$

\section{Introduction}

In organic chemistry, the prefix "homo" (from Ancient Greek "oposs", the same) is used in the nomenclature to characterize a pair of substances which have chemical structures that differ by one methylene $\left(\mathrm{CH}_{2}\right)$ group. This nomenclature is widely applied to amino acids. The most prominent pair is 
L-cysteine and L-homocysteine. Other less widely known pairs of amino acids are L-citrulline and L-homocitrulline, and the tripeptides glutathione and homoglutathione due to its central L-homocysteine moiety. "Homo" amino acids should not be confused with "iso" amino acids, such as L-leucine and L-isoleucine, which are isomeric and have the same molecular mass. Another "homo" amino acid pair is L-arginine (Arg) and L-homoarginine (hArg) (Fig. 1). In chemistry, hArg has been known for at least a century. Yet, the scientific interest in this amino acid has been marginal until recently. hArg has been even believed for at least 50 years to be an exogenous compound with no significance at all for humans or animals. The primary interests in hArg were, therefore, its use as an internal standard for the HPLC analysis of other Arg metabolites, including asymmetric dimethylarginine (ADMA) and symmetric dimethylarginine (SDMA), and in association with the guanidination reaction for determining reactive lysine, bioavailable lysine, and gut endogenous lysine (Rutherfurd 2015; Yin et al. 2015). Yet, things changed only very recently, when hArg was measured in human blood and its concentration was found to correlate with functional parameters, suggesting potential physiological functions in humans (Valtonen et al. 2008). Of course, by analogy, hArg has also been tested as a potential substrate for nitric oxide synthase (NOS) (Hecker et al. 1991; Moali et al. 1998), which catalyzes the conversion of Arg to nitric oxide (NO) and L-citrulline. NO is one of the smallest signaling molecules and possesses several biological functions in plants and animals, including humans. The potential utility of hArg as a substrate for NOS has early led to the assumption that, if hArg would have a biological role, that would be due to its function as an NO precursor. Likely, this assumption has initiated many experimental and clinical studies, which eventually revealed hArg, specifically at concentrations lower than average concentrations measured in healthy humans, as a novel cardiovascular risk factor (März et al. 2010; Pilz et al. 2011a, b; Pilz et al. 2014; Tomaschitz et al. 2015). This is in contrast to the use of higher concentrations of ADMA, the asymmetrically guanidine $\left(N^{\mathrm{G}}\right)$-dimethylated Arg derivative, as a biomarker for risk of cardiovascular
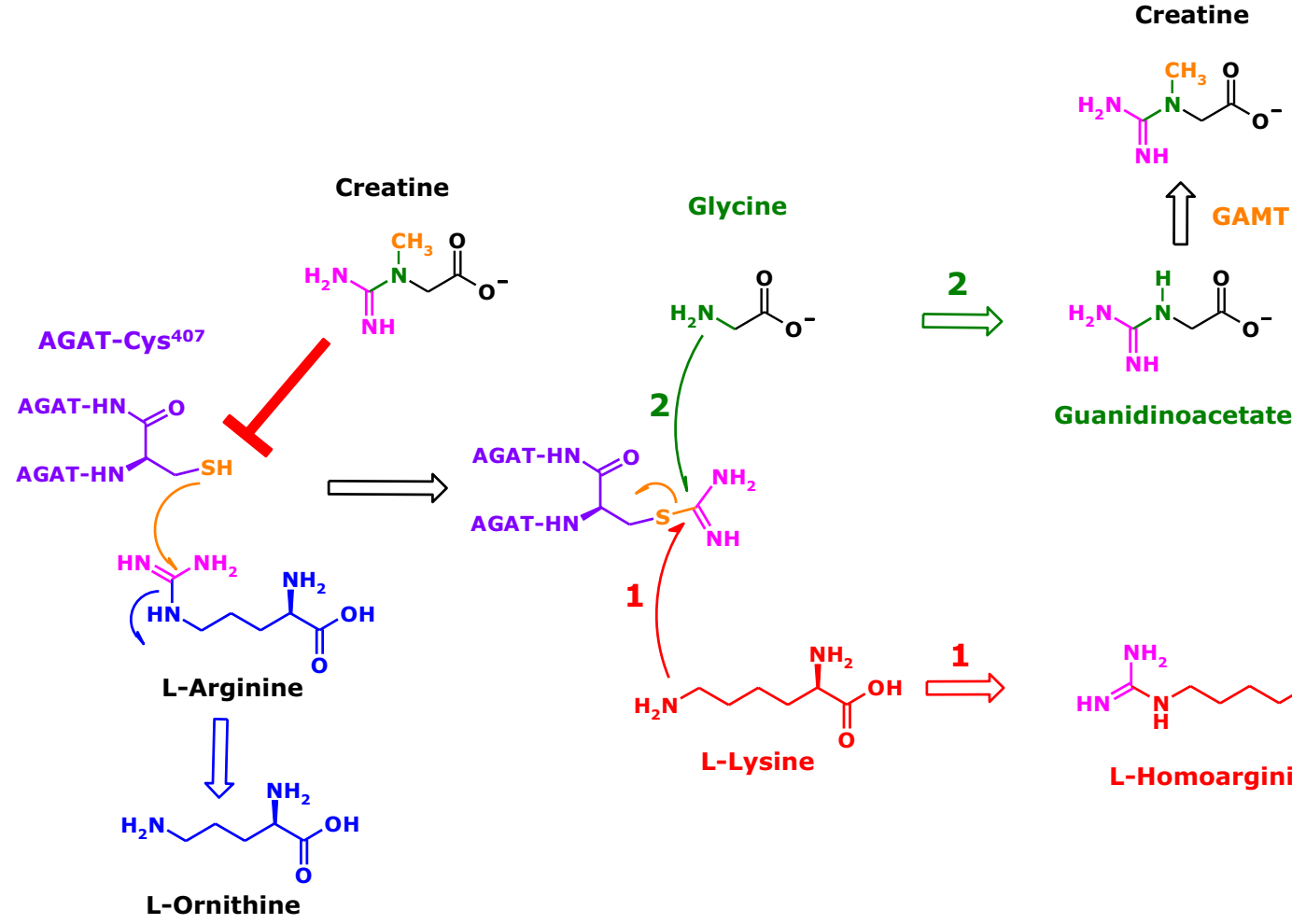

Guanidinoacetate<smiles>N=C(N)NCCCCC(N)C(=O)O</smiles>

L-Homoarginine

Fig. 1 Proposed mechanisms for the biosynthesis of L-homoarginine and guanidinoacetate from L-arginine. Arginine:glycine amidinotransferase (AGAT) catalyzes the formation of guanidinoacetate from L-arginine and glycine, with L-ornithine being a co-product (Humm et al. 1997). The mechanism has been proposed to include nucleophilic attach of the sulfhydryl ( $\mathrm{SH})$ group of Cys407 of the recombinant human AGAT (AGAT-Cys ${ }^{407}$ ) to form a thiocarbamide derivative stabilized by Asp170 and Asp305 (Humm et al. 1997). Nucleophilic attack of the amino group of glycine on the $\mathrm{C}$ atom of the thiocar- bamide moiety releases guanidinoacetate (reaction 2). AGAT-catalyzed L-homoarginine synthesis by such a mechanism would require nucleophilic attack of the terminal amino group of L-lysine on the $\mathrm{C}$ atom of the thiocarbamide moiety (reaction 1). Guanidinoacetate methyltransferase (GAMT) methylates guanidinoacetate to form creatine. Creatine inhibits AGAT-catalyzed synthesis of L-homoarginine in vivo in mice (Choe et al. 2013; Kayacelebi et al. 2015b), presumably by reacting with the $\mathrm{SH}$ group of AGAT-Cys ${ }^{407}$ 
disorders. ADMA is generally believed to exclusively exert its biological actions by inhibiting NOS-catalyzed synthesis of NO from Arg. Considerably lower circulating concentrations of hArg in blood and lower affinity of hArg to several NOS isoforms, as compared to Arg, challenge the current general belief that NO produced from hArg is the sole mechanism that explains the recently discovered pathophysiological functions of hArg. There are also doubts that the inhibitory action of ADMA on NO synthesis is exclusively responsible for the cardiovascular risk of ADMA. The very weak inhibitory potency of ADMA towards endothelial NOS $\left(\mathrm{IC}_{50} 12 \mu \mathrm{M}\right.$; Kielstein et al. 2007) is the strongest argument that challenges the still prevailing dogma of ADMA's principal action in the renal and cardiovascular systems.

In consideration of the emerging pathophysiological importance of hArg, as revealed by the numerous scientific reports of the last few years, the almost completely unknown underlying mechanisms for the actions of hArg, the incompletely understood or explained effects of ADMA in biological systems, together with the fact that Arg is the common precursor of hArg and ADMA, we thought that the time was ripe for the publication of a special issue in the journal Amino Acids just devoted to hArg, Arg and their relatives including ADMA and SDMA. We are very pleased to present to the readers the special issue in hand which we introduce to the readership in brief in the following sections.

Analytical methods for the qualitative and quantitative analysis of native and modified amino acids, peptides and proteins in biological samples are indispensable tools for understanding their multiple roles in living organisms, as well as for the development of strategies for reliable diagnosis and successful therapy of particular diseases. From an analytical point of view, amino acids and their metabolites may represent big challenges for particular techniques of separation and detection. Difficulties result from the relatively large number of amino acids and their diversity with respect to chemical structure and physicochemical properties (including stability), as well as from the occurrence at greatly differing concentrations in many cases. Thus, specific analysis of hArg requires its separation not only from Arg that coexists in large molar excess over hArg, but also from other coexisting Arg derivatives such as the $N^{\mathrm{G}}$-methylated ADMA, SDMA and monomethyl arginine (MMA), as well as from other endogenous isobaric amino acids, notably trimethyllysine. By means of convenient HPLC with fluorescence detection after pre-column derivatization with $o$-phthaldialdehyde (OPA) and thiols such as $\mathrm{N}$-acetylcysteine, in principle, all primary amino acids including hArg can be reliably determined in biological samples (Hou et al. 2015). Hyphenated techniques, notably the couple of mass spectrometry (MS) with liquid chromatography (LC) (i.e., LC-MS and LC-MS/MS) or gas chromatography (i.e., GC-MS, GC-MS/MS), allow not only for highly accurate measurement of these and other amino acids, but they are a unique analytical tool to study biosynthesis and metabolism of amino acids in complex biological samples such as plasma and urine (Cordts et al. 2015; Kayacelebi et al. 2015a, b). Commercially available immunological methods such as ELISA are in general very popular. ELISA is also widely used for the measurement of ADMA and hArg in clinical studies. These assays need validation by other more reliable methods such as LC-MS/ MS and GC-MS/MS. Yet, one may expect diverging quantitative results (Cordts et al. 2015).

In adults, elevated concentrations of ADMA and SDMA, as well as reduced concentrations of hArg, are generally accepted cardiovascular risk factors. Recent clinical investigations on these Arg metabolites in adults extend their key importance to other diseases in adults, including multiple sclerosis, chronic liver and kidney disease, obesity, rheumatoid arthritis, coronary artery disease, and peripheral arterial occlusive disease (Dimitroulas et al. 2015; Frenay et al. 2015a, b; Haghikia et al. 2015; Kayacelebi et al. 2015c; Kruszelnicka et al. 2015; May et al. 2015; Pilz et al. 2015a; Schneider et al. 2015; Sitar et al. 2015). In childhood and adolescence, the significance of ADMA or hArg as cardiovascular risk factors is little investigated and incompletely understood. The circulating concentration of ADMA in healthy children is more than two times higher than in healthy adults (Lücke et al. 2007), suggesting that ADMA and presumably the whole Arg/NO pathway greatly differ between children/adolescents and adults, i.e., that children are not small adults. In the present work, emphasis was given to the Arg/NO pathway, including hArg in healthy children and adolescents, as well as in those suffering from type 1 diabetes mellitus, Duchenne muscular dystrophy, and growth hormone deficiency (GHD) (Carmann et al. 2015; Hörster et al. 2015; Langen et al. 2015; see also Thum et al. 2007).

In a mice model of stroke, oral administration of hArg has been demonstrated to restrict the seizure area. Interestingly, hArg supplementation was found to reduce blood glucose concentration in diet-induced obese mice (Stockebrand et al. 2015). These are promising observations, indicating a potential for pharmacological use of hArg in particular diseases, such as obesity and diabetes. In a rat model of acutely hyperhomocysteinemia-induced endothelial dysfunction, the role that ADMA and SDMA was investigated (Magné et al. 2015). Methionine load induced a sustained increase in total homocysteine and a decrease in vascular reactivity. The absence of an elevation of circulating ADMA and SDMA in this model suggested that endothelial dysfunction induced by acute hyperhomocysteinemia may be explained by a down-regulation of the proteasome (Magné et al. 2015). 
Previous animal and human (Bode-Böger 2006) studies indicated that short-term oral administration of Arg, the common precursor of hArg, ADMA and SDMA, at daily doses up to $10 \mathrm{~g}$ is safe. Long-term oral administration of Arg supports the pharmacological safety of Arg in animals and humans (Kayacelebi et al. 2015b; Yang et al. 2015). Also, acute intravenous administration of large amounts of Arg, as performed in the so called Arginine Test which is used clinically in the diagnosis of GHD, underlines the pharmacological safety of Arg even in children (Hu et al. 2015; Kayacelebi et al. 2015b). Interestingly, acute and long-term oral administration of Arg has different effects with respect to hArg and ADMA than acute intravenous administration of high doses of Arg. Benefits from pharmacological or supplemental use of Arg are expected to be more than enhancement of NO synthesis by increasing the substrate availability of Arg (Wu et al. 2009). Potential benefits of oral administration of Arg could be saving nitrite bioavailability due to improved reabsorption of nitrite in the proximal tubule of the nephron (Schneider et al. 2015), as nitrite is the major NO reservoir (Gladwin et al. 2006), and due to enhanced hArg biosynthesis in comparison to ADMA (Kayacelebi et al. 2015b). This may be of particular importance because hArg and ADMA seem to act antagonistically at least in the circulatory system (Tsikas and Kayacelebi 2014).

Although the first report on the biological importance of hArg appeared only very recently (Valtonen et al. 2008), hArg attracts the increasing attention of scientists from various disciplines, and the number of scientific reports dealing with hArg increases exponentially. The current state of the pathophysiology of hArg and the $N^{\mathrm{G}}$-methylated Arg analogs ADMA and SDMA in the circulation and in some organs (including the brain) in conditions such as pregnancy is thoroughly reviewed and discussed in the present special issue (Bernstein et al. 2015; Khalil et al. 2015; Papageorgiou et al. 2015; Pilz et al. 2015b). The transport of ornithine (a product of Arg hydrolysis by the cytosolic arginase I and the mitochondrial arginase II) and related basic amino acids was also reviewed and discussed in relation to Arg and hArg homeostasis in cells and the whole body (Monné et al. 2015; Schlune et al. 2015). Arg has been reported to serve as a substrate for an endothelial-like NOS in platelets and erythrocytes. Yet, proteomic studies indicated that human platelets lack any NOS proteins, and measurement of NOS activity in stimulated and non-stimulated platelets by a sensitive and specific stable-isotope dilution GC-MS method failed to detect any NOS activity (for a review see Gambaryan and Tsikas 2015).

Our knowledge of the physiological and pathological importance of hArg grew enormously in the last few years. Nevertheless, there is a deep knowledge gap with respect to the basic aspects of hArg. These issues include the biosynthesis and metabolism of hArg. There is strong evidence from in vitro and in vivo animal experiments (Choe et al. 2013) and valuable information from very few human individuals (Davids et al. 2012) that arginine:glycine amidinotransferase (AGAT; EC 2.1.4.1) is the enzyme that is mainly responsible for the synthesis of hArg. Yet, the precise mechanism of this biosynthetic route and the factors which may modulate this reaction are essentially unknown. Actually, human AGAT catalyzes the synthesis of guanidinoacetate from L-arginine and glycine, thereby generating L-ornithine (Fig. 1). The commercial inaccessibility of isolated and purified AGAT or recombinant AGAT preparations is a major limitation in the current research of the biochemistry of hArg. Animal experiments indicate that the AGAT route is not the only pathway by which hArg is biosynthesized from Arg and L-lysine. These potentially alternative mechanisms remain to be elucidated. hArg has been reported to serve as a substrate for several NOS isoforms (Hecker et al. 1991; Moali et al. 1998). The function of hArg as a substrate for NOS demands deeper investigations using pure enzyme preparations, including recombinant enzymes and specific measurement of $\mathrm{NO}$ and its metabolites nitrite and nitrate. Guanidine $-{ }^{15} \mathrm{~N}$ labeled hArg would be the most suitable substrate for future investigations to answer open questions regarding the function of hArg as a precursor of NO. Besides serving as a substrate for NOS, hArg seems to act differently with regard to oxidative stress in vitro in erythrocytes and organs of the rat, albeit at supraphysiological concentrations (Sasso et al. 2015). Analogous to Arg, yet at a much lower concentration, one may suppose that hArg may participate in many of those pathways in which Arg is involved. This is worth of investigation in forthcoming studies.

Closing the Editorial, we would like to thank Prof. Gert Lubec for giving us the opportunity to organize this special issue for Amino Acids and for his great support and guidance of editing. We also would like to thank the authors and their coworkers for contributing to this special issue. We are grateful to the reviewers who spent their valuable time to improve the quality of the papers included in this work.

Acknowledgments Work in the authors' laboratories was supported by a Grant (TS 60/4-1) from the Deutsche Forschungsgemeinschaft (DFG) at the Hannover Medical School (Hannover, Germany), and by Agriculture and Food Research Initiative Competitive Grants from the USDA National Institute of Food and Agriculture, International Council of Amino Acid Science, the National Natural Science Foundation of China, and Texas A\&M AgriLife Research.

\section{Compliance with ethical standards}

Conflict of interest The authors declare no conflict of interests.

Ethical statement In this work no animal or human studies were performed. 


\section{References}

Bernstein HG, Jäger K, Dobrowolny H, Steiner J, Keilhoff G, Bogerts B, Laube G (2015) Possible sources and functions of L-homoarginine in the brain: review of the literature and own findings. Amino Acids. doi:10.1007/s00726-015-1960-y

Bode-Böger SM (2006) Effect of arginine supplementation on NO production in man. Eur J Clin Pharmacol 62:91-99

Carmann C, Lilienthal E, Weigt-Usinger K, Schmidt-Choudhury A, Hörster I, Kayacelebi AA, Beckmann B, Chobanyan-Jürgens K, Tsikas D, Lücke T (2015) The L-arginine/NO pathway, homoarginine, and nitrite-dependent renal carbonic anhydrase activity in young people with type 1 diabetes mellitus. Amino Acids. doi:10.1007/s00726-015-2027-9

Choe CU, Atzler D, Wild PS, Carter AM, Boger RH, Ojeda F et al (2013) Homoarginine levels are regulated by arginine:glycine amidinotransferase and affect stroke outcome: results from human and murine studies. Circulation 128:1451-1461

Cordts K, Atzler D, Qaderi V, Sydow K, Böger RH, Choe CU, Schwedhelm E (2015) Measurement of homoarginine in human and mouse plasma by LC-MS/MS and ELISA: a comparison and a biological application. Amino Acids. doi:10.1007/ s00726-015-2037-7

Davids M, Ndika JD, Salomons GS, Blom HJ, Teerlink T (2012) Promiscuous activity of arginine:glycine amidinotransferase is responsible for the synthesis of the novel cardiovascular risk factor homoarginine. FEBS Lett 586:3653-3657

Dimitroulas T, Hodson J, Sandoo A, Smith J, Kitas GD (2015) Symmetric dimethylarginine (SDMA) serum levels in rheumatoid arthritis: correlations with insulin resistance and disease activity scores. Amino Acids. doi:10.1007/s00726-015-1953-x

Frenay AS, van den Berg E, de Borst MH, Beckmann B, Tsikas D, Feelisch M, Navis G, Bakker SJL, van Goor H (2015a) Plasma ADMA associates with all-cause mortality in renal transplant recipients. Amino Acids. doi:10.1007/s00726-015-2023-0

Frenay AS, Kayacelebi AA, van den Berg E, de Borst MH, Beckmann B, van Goor H, Bakker SJL, Tsikas D (2015b) High urinary homoarginine excretion is associated with low rates of all-cause mortality and graft failure in renal transplant recipients. Amino Acids. doi:10.1007/s00726-015-2038-6

Gambaryan S, Tsikas D (2015) A review and discussion of platelet nitric oxide and nitric oxide synthase: do blood platelets produce nitric oxide from L-arginine or nitrite? Amino Acids. doi:10.1007/s00726-015-1986-1

Gladwin MT, Raat NJ, Shiva S, Dezfulian C, Hogg N, Kim-Shapiro DB, Patel RP (2006) Nitrite as a vascular endocrine nitric oxide reservoir that contributes to hypoxic signaling, cytoprotection, and vasodilation. Am J Physiol Heart Circ Physiol 291:H2026-2035

Haghikia A, Kayacelebi AA, Beckmann B, Hanff E, Gold R, Haghikia A, Tsikas D (2015) Serum and cerebrospinal fluid concentrations of homoarginine, arginine, asymmetric and symmetric dimethylarginine, nitrite and nitrate in patients with multiple sclerosis and neuromyelitis optica. Amino Acids. doi:10.1007/ s00726-015-2015-0

Hecker M, Walsh DT, Vane JR (1991) On the substrate specificity of nitric oxide synthase. FEBS Lett 294:221-224

Hörster I, Weigt-Usinger K, Carmann C, Chobanyan-Jürgens K, Köhler C, Schara U, Kayacelebi AA, Beckmann B, Tsikas D, Lücke T (2015) The L-arginine/NO pathway and homoarginine are altered in Duchenne muscular dystrophy and improved by glucocorticoids. Amino Acids. doi:10.1007/s00726-015-2018-x

Hou Y, Jia S, Nawaratna G, Hu S, Dahanayaka S, Bazer FW, Wu G (2015) Analysis of L-homoarginine in biological samples by HPLC involving precolumn derivatization with $o$-phthalaldehyde and $N$-acetyl-L-cysteine. Amino Acids. doi:10.1007/s00726-015-1962-9

Hu SD, Li XL, Rezaei R, Meininger CJ, McNeal CJ, Wu G (2015) Safety of long-term dietary supplementation with L-arginine in pigs. Amino Acids 47:925-936

Humm A, Fritsche E, Steinbacher S, Huber R (1997) Crystal strukture and mechanism of human L-arginine:glycine amidinotransferase: a mitochondrial enzyme involved in creatine biosynthesis. EMBO J 16:3373-3385

Kayacelebi AA, Knöfel AK, Beckmann B, Hanff E, Warnecke G, Tsikas D (2015a) Measurement of unlabeled and stable isotope-labeled homoarginine, arginine and their metabolites in biological samples by GC-MS and GC-MS/MS. Amino Acids. doi:10.1007/s00726-015-1984-3

Kayacelebi AA, Langen J, Weigt-Usinger K, Chobanyan-Jürgens K, Mariotti F, Schneider JY, Rothmann S, Frölich JC, Atzler D, Choe CU, Schwedhelm E, Huneau JF, Lücke T, Tsikas D (2015b) Biosynthesis of homoarginine (hArg) and asymmetric dimethylarginine (ADMA) from acutely and chronically administered free L-arginine in humans. Amino Acids. doi:10.1007/ s00726-015-2012-3

Kayacelebi AA, Willers J, Pham VV, Hahn A, Schneider JY, Rothmann S, Frölich JC, Tsikas D (2015c) Plasma homoarginine, arginine, asymmetric dimethylarginine and total homocysteine interrelationships in rheumatoid arthritis, coronary artery disease and peripheral artery occlusion disease. Amino Acids. doi:10.1007/s00726-015-1915-3

Khalil A, Hardman L, O`Brien P (2015) The role of arginine, homoarginine and nitric oxide in pregnancy. Amino Acids. doi:10.1007/ s00726-015-2014-1

Kielstein A, Tsikas D, Galloway GP, Mendelson JE (2007) Asymmetric dimethylarginine (ADMA) - a modulator of nociception in opiate tolerance and addiction? Nitric Oxide 17:55-59

Kruszelnicka O, Chyrchel B, Golay A, Surdacki A (2015) Differential associations of circulating asymmetric dimethylarginine and cell adhesion molecules with metformin use in patients with type 2 diabetes mellitus and stable coronary artery disease. Amino Acids. doi:10.1007/s00726-015-1976-3

Langen J, Kayacelebi AA, Beckmann B, Weigt-Usinger K, Carmann C, Hörster I, Lilienthal E, Richter-Unruh A, Tsikas D, Lücke $\mathrm{T}$ (2015) Homoarginine (hArg) and asymmetric dimethylarginine (ADMA) in short stature children without and with growth hormone deficiency: hArg and ADMA are involved differently in growth in the childhood. Amino Acids. doi:10.1007/ s00726-015-2028-8

Lücke T, Kanzelmeyer N, Kemper MJ, Tsikas D, Das AM (2007) Developmental changes in the L-arginine/nitric oxide pathway from infancy to adulthood: plasma asymmetric dimethylarginine levels decrease with age. Clin Chem Lab Med 45:1525-1530

Magné J, Huneau JF, Borderie D, Mathé V, Bos C, Mariotti F (2015) Plasma asymmetric and symmetric dimethylarginine in a rat model of endothelial dysfunction induced by acute hyperhomocysteinemia. Amino Acids. doi:10.1007/s00726-015-1959-4

März W, Meinitzer A, Drechsler C, Pilz S, Krane V, Kleber ME, Fischer J, Winkelmann BR, Böhm BO, Ritz E, Wanner C (2010) Homoarginine, cardiovascular risk, and mortality. Circulation 122:967-975

May M, Kayacelebi AA, Batkai S, Jordan J, Tsikas D, Engeli S (2015) Plasma and tissue homoarginine concentrations in healthy and obese humans. Amino Acids. doi:10.1007/s00726-015-1922-4

Moali C, Boucher JL, Sari MA, Stuehr DJ, Mansuy D (1998) Substrate specificity of NO synthases: detailed comparison of L-arginine, homo-L-arginine, their $\mathrm{N}$ omega-hydroxy derivatives, and N omega-hydroxynor-L-arginine. Biochemistry 37:10453-10460

Monné M, Miniero DV, Daddabbo L, Palmieri L, Porcelli V, Palmieri F (2015) Mitochondrial transporters for ornithine and 
related amino acids: a review. Amino Acids. doi:10.1007/ s00726-015-1990-5

Papageorgiou N, Androulakis E, Papaioannou S, Antoniades C, Tousoulis D (2015) Homoarginine in the shadow of asymmetric dimethylarginine: from nitric oxide to cardiovascular disease. Amino Acids. doi:10.1007/s00726-015-2017-y

Pilz S, Meinitzer A, Tomaschitz A, Drechsler C, Ritz E, Krane V, Wanner C, Böhm BO, März W (2011a) Low homoarginine concentration is a novel risk factor for heart disease. Heart 97:1222-1227

Pilz S, Tomaschitz A, Meinitzer A, Drechsler C, Ritz E, Krane V, Wanner C, Böhm BO, März W (2011b) Low serum homoarginine is a novel risk factor for fatal strokes in patients undergoing coronary angiography. Stroke 42:1132-1134

Pilz S, Teerlink T, Scheffer PG, Meinitzer A, Rutters F, Tomaschitz A, Drechsler C, Kienreich K, Nijpels G, Stehouwer CD, März W, Dekker JM (2014) Homoarginine and mortality in an older population: the Hoorn study. Eur J Clin Invest 44:200-208

Pilz S, Putz-Bankuti C, Meinitzer A, März W, Kienreich K, Stojakovic T, Pieber TR, Stauber RE (2015a) Association of homoarginine and methylarginines with liver dysfunction and mortality in chronic liver disease. Amino Acids. doi:10.1007/ s00726-015-2000-7

Pilz S, Meinitzer A, Gaksch M, Grübler M, Verheyen N, Drechsler C, Hartaigh BÓ, Lang F, Alesutan I, Voelkl J, März W, Tomaschitz A (2015b) Homoarginine in the renal and cardiovascular systems. Amino Acids. doi:10.1007/s00726-015-1993-2

Rutherfurd SM (2015) Use of the guanidination reaction for determining reactive lysine, bioavailable lysine and gut endogenous lysine. Amino Acids. doi:10.1007/s00726-015-2007-0

Sasso S, Dalmedico L, Magro DD, Pereira EM, Wyse AT, de Lima DD (2015) Differential in vitro effects of homoarginine on oxidative stress in plasma, erythrocytes, kidney and liver of rats in the absence and in the presence $\alpha$-tocopherol, ascorbic acid or L-NAME. Amino Acids. doi:10.1007/s00726-015-1973-6

Schlune A, vom Dahl S, Häussinger D, Ensenauer R, Mayatepek E (2015) Hyperarginemia due to arginase I deficiency: the original patients and their natural history, and a review of the literature. Amino Acids. doi:10.1007/s00726-015-2032-z

Schneider JY, Rothmann S, Schröder F, Langen J, Lücke T, Mariotti F, Huneau JF, Frölich JC, Tsikas D (2015) Effects of chronic oral L-arginine administration on the L-arginine/NO pathway in patients with peripheral arterial occlusive disease or coronary artery disease: L-Arginine prevents renal loss of nitrite, the major NO reservoir. Amino Acids. doi:10.1007/s00726-015-2031-0

Sitar ME, Kayacelebi AA, Beckmann B, Kielstein JT, Tsikas D (2015) Asymmetric dimethylarginine (ADMA) in human blood: effects of extended haemodialysis in the critically ill patient with acute kidney injury, protein binding to human serum albumin and proteolysis by thermolysin. Amino Acids. doi:10.1007/ s00726-015-1991-4

Stockebrand M, Hornig S, Neu A, Atzler D, Cordts K, Böger RH, Isbrandt D, Schwedhelm E, Choe C (2015) Homoarginine supplementation improves blood glucose in diet-induced obese mice. Amino Acids. doi:10.1007/s00726-015-2022-1

Thum T, Fleissner F, Klink I, Tsikas D, Jakob M, Bauersachs J, Stichtenoth DO (2007) Growth hormone treatment improves markers of systemic nitric oxide bioavailability via insulin-like growth factor-I. J Clin Endocrinol Metab 92:4172-4179

Tomaschitz A, Verheyen N, Gaksch M, Meinitzer A, Pieske B, Kraigher-Krainer E, Colantonio C, März W, Schmidt A, Belyavskiy E, Rus-Machan J, van Ballegooijen AJ, Stiegler C, Amrein K, Ritz E, Fahrleitner-Pammer A, Pilz S (2015) Homoarginine in patients with primary hyperparathyroidism. Am J Med Sci 349:306-311

Tsikas D, Kayacelebi AA (2014) Do homoarginine and asymmetric dimethylarginine act antagonistically in the cardiovascular system? Circ J 78:2094-2095

Valtonen P, Laitinen T, Lyyra-Laitinen T, Raitakari OT, Juonala M, Viikari JS, Heiskanen N, Vanninen E, Punnonen K, Heinonen S (2008) Serum L-homoarginine concentration is elevated during normal pregnancy and is related to flow-mediated vasodilatation. Circ J 72:1879-1884

Wu G, Bazer FW, Davis TA, Kim SW, Li P, Marc Rhoads J, Carey Satterfield M, Smith SB, Spencer TE, Yin Y (2009) Arginine metabolism and nutrition in growth, health and disease. Amino Acids 37:153-168

Yang Y, Wu Z, Jia S, Dahanayaka S, Feng S, Meininger CJ, McNeal CJ, Wu G (2015) Safety of long-term dietary supplementation with L-arginine in rats. Amino Acids. doi:10.1007/ s00726-015-1992-3

Yin J, Ren W, Hou Y, Wu M, Xiao H, Duan J, Zhao Y, Li T, Yin Y, Wu G, Nyachoti CM (2015) Use of homoarginine for measuring true ileal digestibility of amino acids in food protein. Amino Acids. doi:10.1007/s00726-015-1958-5 